Editorial

\title{
Review of risk communication and education strategies around food hygiene and safety for children and young people
}

\section{A B S T R A C T}

This article has been developed to investigate successful methods of risk communication and education of young people on food hygiene and safety. The authors operate the e-Bug project, an international programme consisting of educational materials for schools and communities covering health protection topics including infection prevention and antibiotic resistance. This review will help inform SafeConsumE, a Horizon2020 funded project that will involve the development of new education materials for young people with the aim to prevent foodborne illness that align with relevant methods of risk communication.

\section{Introduction}

Foodborne illnesses are common across the world and can be a serious threat to life. There are more than 200 different diseases that can be spread through food, and vulnerable groups such as children and the elderly are more at risk. The World Health Organisation (WHO) estimates that 23 million people suffer from foodborne illnesses each year in Europe, causing 5000 deaths annually (WHO, 2015). In addition, approximately $30 \%$ of all deaths from foodborne diseases are in children under the age of 5 years (WHO, 2015). There is also growing concern around antimicrobial resistance in foodborne pathogens and WHO lists two foodborne bacteria, Salmonella and Campylobacter as 'High priority' on the list of pathogens for which new antibiotics are urgently needed (WHO, 2017). This highlights the potential severity of infections transmitted through food, and also the importance of risk communication and education of the public on food safety and hygiene.

\section{Why educate and communicate risk to young people?}

Risk communication is the mutual sharing of information to improve attitudes and behaviours around potential risk situations. Behaviour change has been linked to individual's perception of their own risk in behaviour models (Ahmed, Naik, Willoughby, \& Edwards, 2012). Young people are an important audience for education on the risk of improper food hygiene and as they tend to be both over-confident in their perception of personal risk and practice more unsafe behaviours (Diplock et al., 2017). By educating children at a young age we can influence future behaviour and habits before they are even formed, setting a solid foundation for healthy behaviours and choices around food. Furthermore, the messages and behaviours around hygiene that children learn in schools are taken home and can influence the behaviour of other family members. Therefore by targeting school students we are reaching a wider audience and setting safe behaviours for life.

\section{Innovative methods of risk communication for children and young people}

Developing an effective method for food safety education requires multiple components (Jacob, Mathiasen, \& Powell, 2010). As outlined by Jacob et al. (2010), communicating microbial food safety hazards requires good understanding of the target audience; creating a consistent and reliable message; being clear with your message and user testing and evaluating your content (Jacob et al., 2010). There are a wide range of methods currently being utilised to communicate food hygiene to children and young people. These include formal lessons delivered in educational settings such as classrooms; educational games; videos and digital media; and multidisciplinary media campaigns.

\section{Classroom-based interventions}

No standard methodology currently exists for the integration of food hygiene and safety into school curricula (Caraher, Wu, \& Seeley, 2010; Egan et al., 2008). However, in the field of hygiene, infection prevention and antibiotic resistance, free educational resources are provided by e-Bug (www.e-bug.eu). e-Bug, operated by Public Health England provides a wide range of resources for use in educational settings, including lesson plans, worksheets, games and interactive activities (Fig. 1).

e-Bug provides a module on food hygiene under the 'Spread of infection' topic targeted at junior students' aged 7-11 years. This is comprised of a teacher lesson plan, worksheets for students and an interactive activity on handing of raw chicken and spread of microbes (Fig. 2). In a survey of 695 educators in England, food hygiene was one of the three highest rated e-Bug modules, with $98 \%$ of educators rating the lesson as excellent or good (Eley, Young, \& McNulty, 2017).

In addition, an evaluation of the e-Bug teaching packs in three European countries found the resources to significantly improve knowledge in young people (Lecky et al., 2010). The study used before, after and knowledge retention questionnaires in control and interventions schools to assess student knowledge gain after receiving e-Bug 


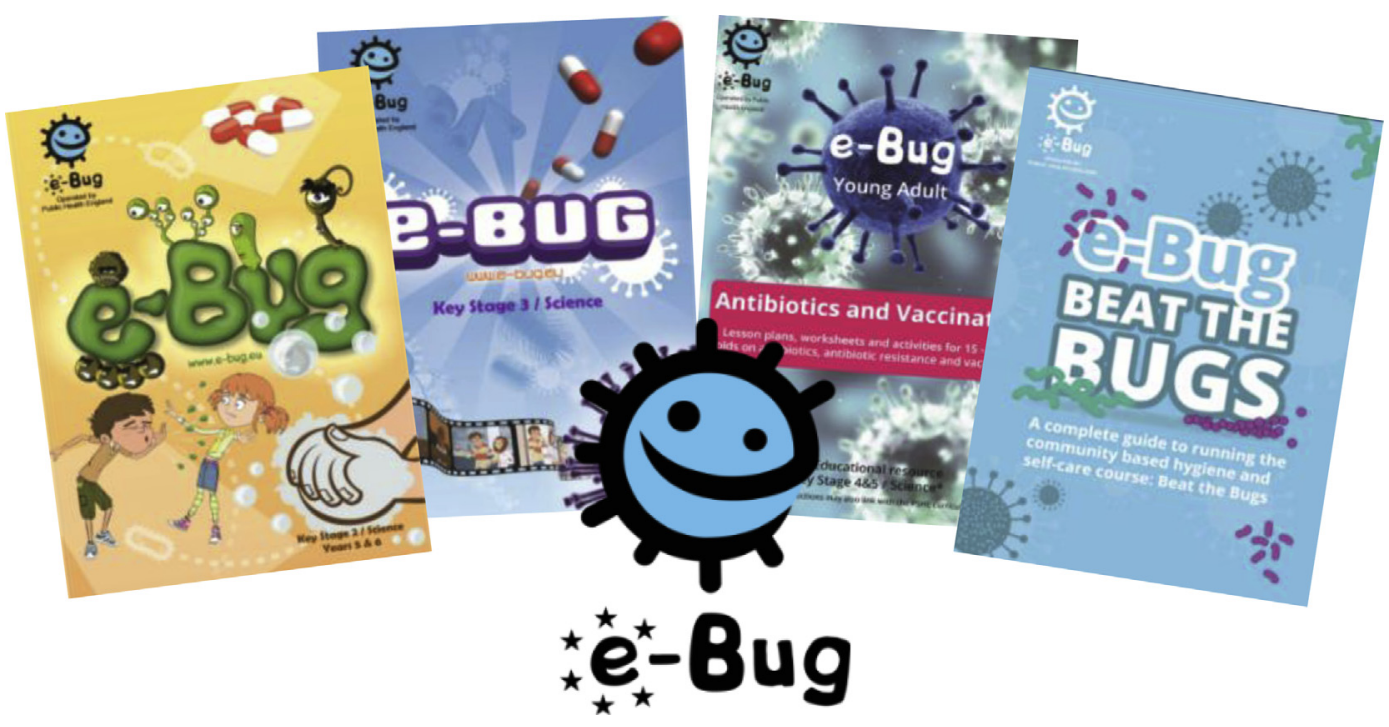

Fig. 1. e-Bug is an international educational program that provides interactive and curriculum aligned lessons on infection prevention, hygiene and antibiotics. e-Bug was developed in 2006 as part of an EU Directorate General for Health and Consumer Protection (DG-SANCO) project, with 18 partner countries across Europe. Since then, e-Bug has expanded in both its available materials and scope and is now available in 23 different languages.

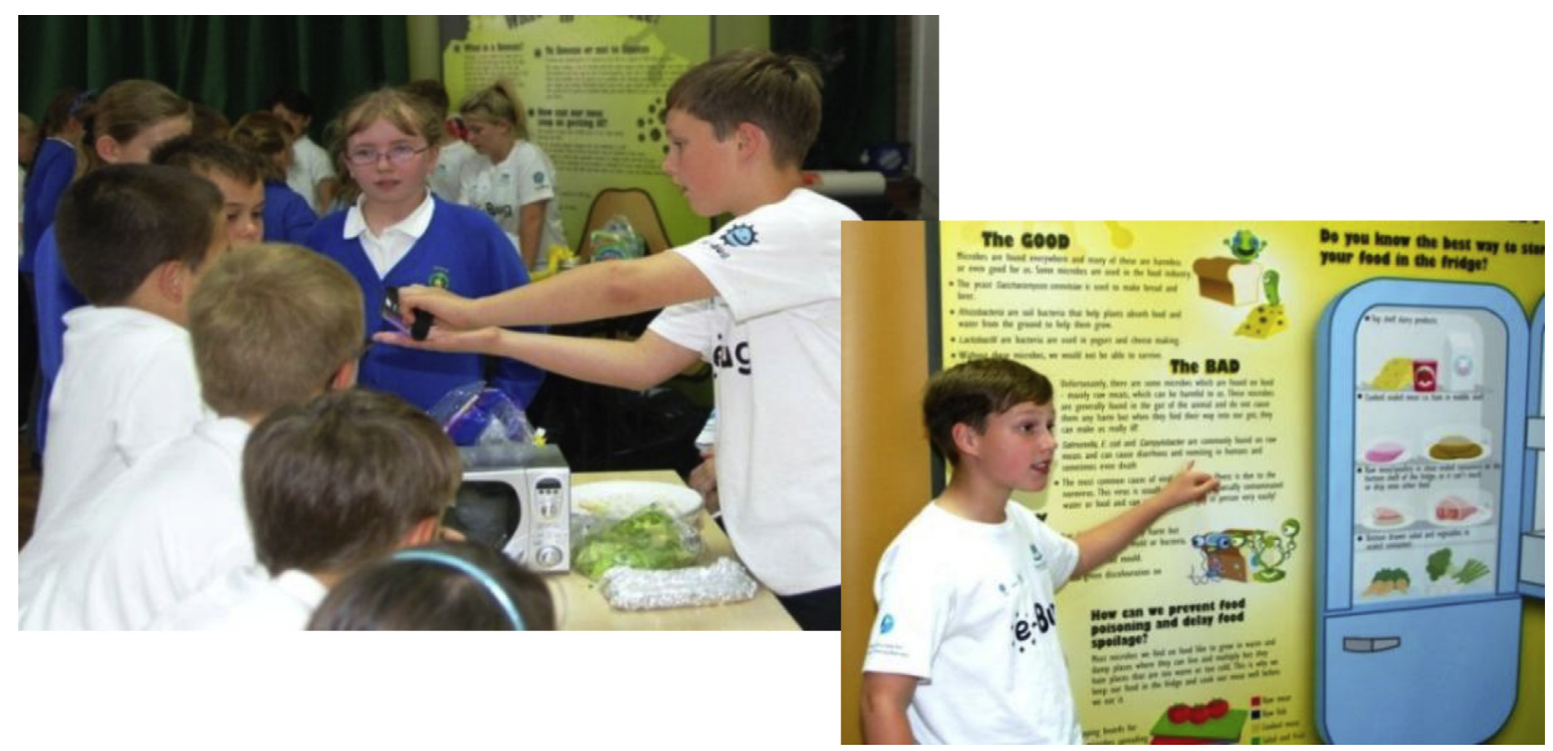

Fig. 2. e-Bug provides an interactive classroom based lesson on food hygiene for 7-11 year olds. Figure shows peer education led delivery of e-Bug food hygiene lesson.

lessons. For the 'Spread of Infection' module, knowledge after teaching improved significantly in all three countries, and this knowledge was retained six weeks post intervention (Lecky et al., 2010). Furthermore, the e-Bug food hygiene activity has been successfully delivered in a science show setting (Lecky, Hawking, Verlander, \& McNulty, 2014), and an evaluation of its delivery via peer education found the activity to significantly improve knowledge (Young et al., 2017) (Fig. 2).

\section{Communicating risk through games}

Game-based learning (GBL) is being used increasingly to educate and engage children and young people on health. It's effectiveness in communicating risk has been documented for sexual health (Artioli, Berta, De Gloria, Pomicino, \& Secco, 2013; LeGrand et al., 2016); obesity and exercise (Göbel, Hardy, Wendel, Mehm, \& Steinmetz, 2010); mental health (Fleming et al., 2017); and antimicrobial prescribing (Castro-Sánchez et al., 2014). Current examples for communication of safe food hygiene practices include Ninja Kitchen, a 15 level web-based game where players are challenged with cooking different meals safely in a diner setting (Quick, Corda, Chamberlin, Schaffner, \& Byrd-Bredbenner, 2013). Players are awarded points if food is prepared safely and lose points if customers develop foodborne illness (Quick et al., 2013). The effectiveness of Ninja Kitchen at teaching key concepts in food hygiene was evaluated with 1268 middle school students who played the game and performed a pre-test, post-test and follow-up test to measure knowledge gain and retention. The game was shown to be effective at teaching and greatest knowledge retention was reported in 'Preventing danger zone hazards'. Chicken Surprise, an online game developed by e-Bug, also teaches on food hygiene by challenging players to make a chicken sandwich safely (Fig. 3). Google analytics of the e-Bug website reported Chicken Surprise as one of the most popular student games (Young et al., 2015). Outside of the school setting, educational games have been used in food industry as a means of training hygiene skills in workers. Serious Gordon, a kitchen role-play game, scores players on their ability to complete cooking and hygiene tasks effectively (i.e. correct hand washing) to progress (Mac Namee 
A

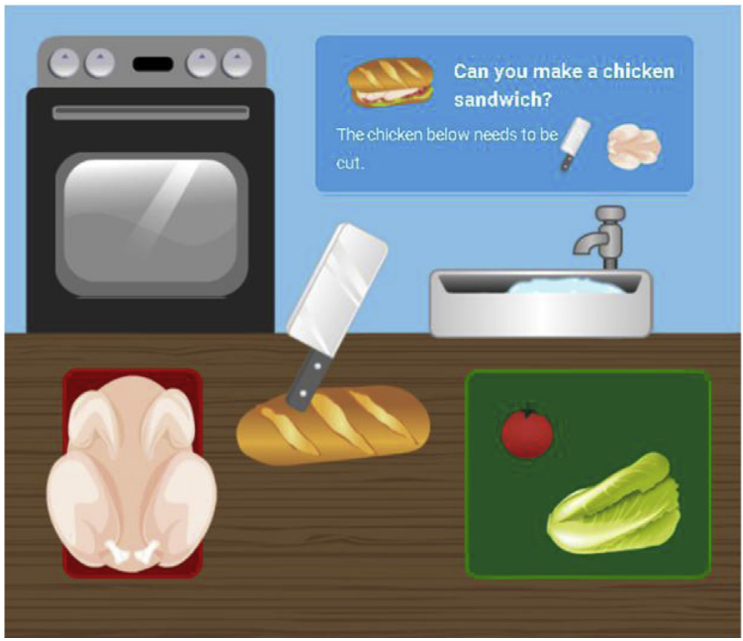

B

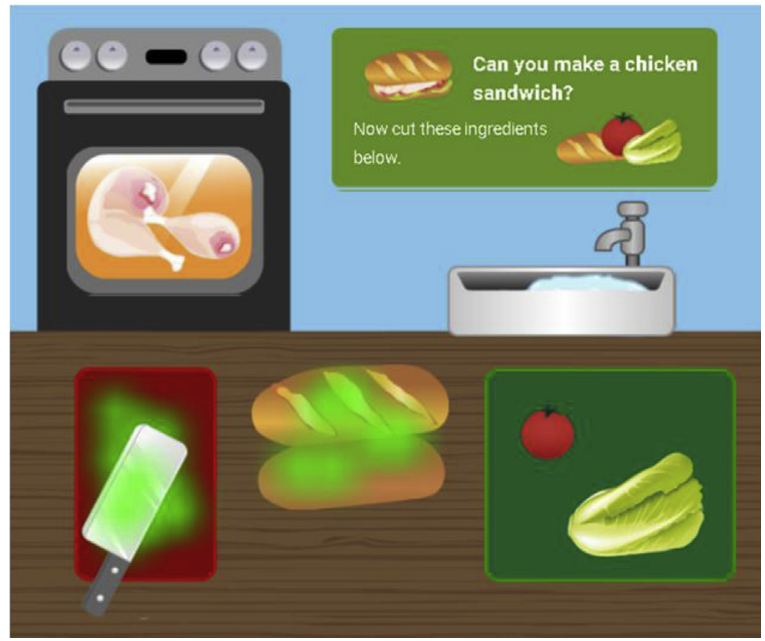

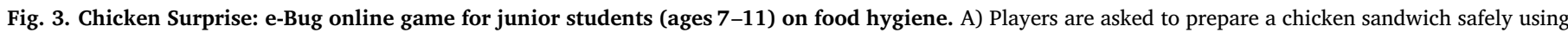
the ingredients and materials provided; B) Bad food hygiene behaviours are showcased through the spread of dangerous microbes.

et al., 2006). An evaluation of the game showed improved knowledge on food hygiene practices and participants found gaming more engaging than traditional textbook learning (Mac Namee et al., 2006).

\section{Digital and social media}

The use of digital and social media websites is one of the most common activities of today's children and adolescents (O'Keeffe \& Clarke-Pearson, 2011). Indeed, these platforms are now a popular tool for dissemination and communication of information in the digital era. For example, a multitude of food safety videos exist on the popular media platform YouTube with their effectiveness shown to correlate with design quality and choice of key messages conveyed (Rhoades \& Ellis, 2010). An innovative example of the use of multiple formats of digital media is Safe Eats, a social media-based intervention designed for young adults to change food hygiene practices (Mayer; Harrison, 2012). The intervention was comprised of regular social media videos that focused on safe preparation of food and motivational posts targeted at undergraduate students. When delivered alongside traditional lectures, students reported that they learned more from the social media intervention. Greatest knowledge gain was reported in students who received both the lecture and social media intervention (Mayer; Harrison, 2012). Utilisation of a multidisciplinary social media intervention was also employed for the "4 Day Throw Away" campaign designed by the University of Nebraska (James, Albrecht, Litchfield, \& Weishaar, 2013). Briefly, the campaign consisted of four YouTube videos (11759 views); Facebook page (21240 post views); Twitter page (51 followers) and was developed from the Health Belief Model (James et al., 2013).

Entertaining media such as musical parodies are also being investigated as useful tools for risk communication. For example, The Food Safety Music website, developed by University of California, Davis, provides an extensive range of contemporary songs re-written to include food safety focused lyrics (Winter et al., 2009). Examples include 'Don't get sicky with it' and 'We are the Microbes' (Winter et al., 2009). Food safety parodies were evaluated with 8-12 year olds enrolled in a youth summer program who participated in weeklong sessions that incorporated numerous songs and involved daily preparation of a meal. Pre- and post-tests showed improved knowledge in all behaviours that ranged from 13 to $29 \%$ in 2004 program and $5-13 \%$ in 2008 program (Winter et al., 2009).

\section{Outlook for risk communication}

Effective risk communication on food hygiene will rely on the use of relevant and accessible methods in the digital era. To this end, e-Bug are currently developing novel food hygiene activities for teenagers, and this will take place as part of the EU SafeConsumE project which runs from 2017 to 2021 and involves 32 partner organisations across 14 countries (www.Safeconsume.eu). SafeConsumE, funded by Horizon2020, aims to change consumer behaviour and reduce the health burden from foodborne illnesses. Through effective communication and educational strategies, we can contribute to reducing foodborne illness in the youngest and most vulnerable members of our society. In doing so we hope to promote better health outcomes and healthier behaviours towards food that can be maintained for life.

\section{References}

Ahmed, H., Naik, G., Willoughby, H., \& Edwards, A. G. (2012). Communicating risk. BMJ, 344, e3996.

Artioli, S., Berta, R., De Gloria, A., Pomicino, A., \& Secco, N. (2013). A serious game to inform about HIV prevention: HInVaders, a case study. Games for health (pp. 3-13). Wiesbaden: Springer Vieweg.

Caraher, M., Wu, M., \& Seeley, A. (2010). Should we teach cooking in schools? A systematic review of the literature of school-based cooking interventions. Journal of the Home Economics Institute of Australia, 17(1), 10-18.

Castro-Sánchez, E., Charani, E., Moore, L., Gharbi, M., \& Holmes, A. (2014). "On call: antibiotics"-development and evaluation of a serious antimicrobial prescribing game for hospital care. Games for health 2014 (pp. 1-7). Wiesbaden: Springer Vieweg.

Diplock, K. J., Jones-Bitton, A., Leatherdale, S. T., Rebellato, S., Dubin, J. A., \& Majowicz, S. E. (2017). Over-confident and under-competent: Exploring the importance of food safety education specific to high school students. Environmental Health Review, 60(3), 65-72.

Egan, M. B., Bielby, G., Eves, A., Lumbers, M. L., Raats, M. M., \& Adams, M. R. (2008) Food hygiene education in UK secondary schools: A nationwide survey of teachers' views. Health Education Journal, 67(2), 110-120.

Eley, C., Young, V., \& McNulty, C. (2017). Beating bugs together. Community Practitioner, 90(3), 42.

Fleming, T. M., Bavin, L., Stasiak, K., Hermansson-Webb, E., Merry, S. N., Cheek, C., Hetrick, S. (2017). Serious games and gamification for mental health: Current status and promising directions. Frontiers in Psychiatry, 7, 215.

Göbel, S., Hardy, S., Wendel, V., Mehm, F., \& Steinmetz, R. (2010). Serious games for health: Personalized exergames. Proceedings of the 18th ACM international conference on multimedia (pp. 1663-1666). ACM.

Jacob, C., Mathiasen, L., \& Powell, D. (2010). Designing effective messages for microbial food safety hazards. Food Control, 21(1), 1-6.

James, K. J., Albrecht, J. A., Litchfield, R. E., \& Weishaar, C. A. (2013). A summative evaluation of a food safety social marketing campaign "4-Day Throw-Away" using traditional and social media. Journal of Food Science Education, 12(3), 48-55.

Lecky, D. M., Hawking, M. K., Verlander, N. Q., \& McNulty, C. A. (2014). Using interactive family science shows to improve public knowledge on antibiotic resistance: Does it work? PLoS One, 9(8), e104556. 
Lecky, D. M., McNulty, C. A., Touboul, P., Herotova, T. K., Beneš, J., Dellamonica, P., et al. (2010). Evaluation of e-Bug, an educational pack, teaching about prudent antibiotic use and hygiene, in the Czech Republic, France and England. Journal of Antimicrobial Chemotherapy, 65(12), 2674-2684.

LeGrand, S., Muessig, K. E., McNulty, T., Soni, K., Knudtson, K., Lemann, A., ... HightowWeidman, L. B. (2016). Epic allies: Development of a gaming app to improve antiretroviral therapy adherence among young HIV-positive men who have sex with men. JMIR Serious Games, 4(1).

Mac Namee, B., Rooney, P., Lindstrom, P., Ritchie, A., Boylan, F., \& Burke, G. (2006). Serious gordon: Using serious games to teach food safety in the kitchen. 9th international conference on computer games. Dublin: Dublin Institute of Technology.

Mayer, A. B., \& Harrison, J. A. (2012). Safe eats: An evaluation of the use of social media for food safety education. Journal of Food Protection, 75(8), 1453-1463.

O'Keeffe, G. S., \& Clarke-Pearson, K. (2011). The impact of social media on children, adolescents, and families. Pediatrics, 127(4), 800-804.

Quick, V., Corda, K. W., Chamberlin, B., Schaffner, D. W., \& Byrd-Bredbenner, C. (2013). Ninja kitchen to the rescue: Evaluation of a food safety education game for middle school youth. British Food Journal, 115(5), 686-699.

Rhoades, E., \& Ellis, J. D. (2010). Food tube: Coverage of food safety issues through video. Journal of Food Safety, 30(1), 162-176.

Winter, C. K., Fraser, A. M., Gleason, J. B., Hovey, S. K., McCurdy, S. M., \& Snider, O. S.
(2009). Food safety education using music parodies. Journal of Food Science Education, 8(3), 62-67.

World Health Organization (2015). WHO estimates of the global burden of foodborne diseases: Foodborne disease burden epidemiology reference group 2007-2015.

World Health Organization (2017). Global priority list of antibiotic-resistant bacteria to guide research, discovery, and development of new antibiotics. Geneva: World Health Organization.

Young, V. L., Cole, A., Lecky, D. M., Fettis, D., Pritchard, B., Verlander, N. Q., ... McNulty, C. A. (2017). A mixed-method evaluation of peer-education workshops for schoolaged children to teach about antibiotics, microbes and hygiene. Journal of Antimicrobial Chemotherapy, 72(7), 2119-2126.

Young, V. L., Rajapandian, V., Eley, C. V., Hoekstra, B. A., Lecky, D. M., \& McNulty, C. A. (2015). Monitoring web site usage of e-bug: A hygiene and antibiotic awareness resource for children. JMIR Research Protocols, 4(4).

Vicki L. Young, Carla L. Brown, Catherine Hayes,

Cliodna A.M. McNulty*

Primary Care Unit, Public Health England, UK

E-mail address: Cliodna.McNulty@phe.gov.uk (C.A.M. McNulty)

* Corresponding author. 